\title{
New evidence in general practice, internal medicine and cardiovascular medicine
}

\author{
Andrea Alberto Conti · Beatrice Dilaghi · \\ Pietro Amedeo Modesti · Carlo Nozzoli
}

Published online: 6 February 2009

(C) SIMI 2009

\section{The opening hours of general practitioners influence the timing in looking for health care by people suffering from minor stroke or transient ischemic attack}

The results of a United Kingdom prospective population based study suggest that the opening hours of general practices influence the behavior of a large number of patients suffering from minor stroke or transient ischemic attack (TIA) occurring out of hours.

Transient ischemic attacks are transient short-lasting neurological events. However, even if the majority of the symptoms of TIAs disappear within $60 \mathrm{~min}$, they can go on up to $24 \mathrm{~h}$. Minor strokes and TIAs are emergencies related to a notable risk of recurrence and recent research indicates that diagnosis and management of minor strokes and TIAs within $24 \mathrm{~h}$ from their insurgence can significantly lower the risk of recurrent stroke.

Nine general practices in the United Kingdom were analysed in the context of a population based prospective

\footnotetext{
A. A. Conti $(\bowtie) \cdot$ P. A. Modesti

Department of Critical Care Medicine and Surgery, University of Florence and Don Carlo Gnocchi Foundation, IRCCS Florence, Viale Morgagni 85,

50134 Florence, Italy

e-mail: aa.conti@dac.unifi.it

B. Dilaghi

Department of Emergency Medicine, Azienda Ospedaliero-Universitaria Careggi,

Florence, Italy

C. Nozzoli

General Medicine Unit,

Azienda Ospedaliero-Universitaria Careggi,

Florence, Italy
}

study, to evaluate the fall out of general practice opening hours on the behavior in looking for health care on the part of individuals suffering from minor stroke or TIA. The study also assessed the sustainability of clinical evaluation within $24 \mathrm{~h}$ from the insurgence of the symptoms. More than 400 (434) subjects with minor stroke and 359 individuals with TIA were considered, and the median time before the call of a general practitioner (GP) in the course of surgery hours was $4 \mathrm{~h}$; approximately two patients out of three $(68 \%)$ with neurological episodes in the course of surgery hours called doctors within $24 \mathrm{~h}$ from the insurgence of symptoms. The median time before the call of the emergency general practitioner service was $1 \mathrm{~h}$, while it was $24.8 \mathrm{~h}$ for subjects waiting to call their registered practice $(p<0.001)$. In individuals who suffered from neurological episodes out of hours, and waiting to contact their GP, the seeking for medical care was inferior for episodes occurring in weekends than in weekdays (odds ratio 0.10). The majority of patients suffering from minor stroke or transient ischemic attack out of hours, in particular in the course of the night or during the weekends, registered a delay in looking for medical care, waiting for the opening hours of their GP. The results of this prospective population based study therefore suggest that the opening hours of general practices influence the behavior of a large number of patients suffering from minor stroke or transient ischemic attack occurring out of hours.

\section{Reference}

Lasserson DS et al (2008) Influence of general practice opening hours on delay in seeking medical attention after transient ischaemic attack (TIA) and minor stroke: prospective population based study. BMJ 337:a1569 
The clinical and public health relevance of the Mediterranean diet

In the primary prevention of relevant chronic diseases Mediterranean-like dietary schemes have a major impact, as emerges from a meta-analysis of prospective cohort studies.

A group of Italian researchers reviewed prospective cohort studies assessing the relationship between the adherence to a Mediterranean diet, the incidence of major chronic pathologies and the mortality in a context of primary prevention. The electronic archives EMBASE, Medline, Web of Science and Cochrane Central Register of Controlled Trials were scanned up to June 2008 to retrieve studies prospectively evaluating the relationship between adherence to a Mediterranean diet, incidence of chronic pathologies and mortality. The authors found 12 studies, including more than $1,500,000$ subjects $(1,574,299$ people) examined and followed for a period between 3 and 18 years. An increase of two points in the adherence to a Mediterranean diet score was significantly associated with a decreased risk in overall mortality (pooled relative risk $0.91,95 \% \mathrm{CI}$ between 0.89 and 0.94). High adherence to a Mediterranean diet proved to have a positive influence on the incidence of or on the mortality resulting from neoplastic diseases (pooled relative risk $0.94,95 \%$ CI between 0.92 and 0.96), on mortality due to cardiovascular causes (pooled relative risk $0.91,95 \%$ CI between 0.87 and 0.95 ) and on the incidence of Alzheimer's disease and Parkinson's disease (pooled relative risk $0.87,95 \% \mathrm{CI}$ between 0.80 and 0.96). In conclusion, a higher adherence to a Mediterranean diet was related to a significant improvement in health conditions, with a significant decrease in general mortality (9\%), in the incidence of or in the mortality due to neoplastic diseases $(6 \%)$, in the mortality because of cardiovascular pathologies (9\%), and in the incidence of Alzheimer's and Parkinson's disease (13\%). These results indicate that, in the primary prevention of relevant chronic diseases, the Mediterranean diet had a major impact, as clearly emerges from a metaanalysis of prospective cohort studies including more than $1,500,000$ people.

\section{Reference}

Sofi F et al (2008) Adherence to Mediterranean diet and health status: meta-analysis. BMJ 337:a1344

\section{Effectiveness of antispasmodics, fibre and peppermint oil in the management of irritable bowel disease}

\footnotetext{
Meta-analysis: antispasmodics, fibre and peppermint oil are effective in the treatment of the irritable bowel syndrome.
}

Irritable bowel disease is a serious syndrome related to gastrointestinal motility disturbance, necessitating both notable comprehension of the nervous system of the gastroenteric system and targeted treatment. To evaluate the effect of antispasmodics, fibre and peppermint oil in the management of irritable bowel disease, a group of North American and European researchers conducted a systematic review with meta-analysis of pertinent and relevant randomised controlled trials retrieved by exploring Embase, Medline and the Cochrane Controlled Trials Register. Trials comparing antispasmodics, fibre and peppermint oil with no therapy or placebo in individuals affected by irritable bowel disease were candidates for assessment in this systematic review if the length of the treatment performed was no shorter than 7 days and if trials included either an ample evaluation of cure or an improvement of symptomatology, or cure or improvement in pain at the abdominal level. Almost 400 patients (392) were studied in 4 clinical trials comparing treatment with peppermint oil with placebo, and the relative risk of persistent symptomatology resulted 0.43 (95\% CI between 0.32 and 0.59). Almost 600 patients (591) were studied in 12 clinical trials comparing treatment with fibre with no treatment or placebo, and the relative risk of persistent symptomatology was 0.87 (95\% CI between 0.76 and 1.00). Almost 1,800 patients $(1,778)$ were studied in 22 clinical trials comparing treatment with antispasmodics with placebo, and the relative risk of persistent symptoms was 0.68 (95\% CI between 0.57 and 0.81). Among the antispasmodics analysed hyoscine (in 426 subjects in 3 trials; relative risk $0.63,95 \%$ CI $0.51-0.78$ ) and otilonium (in 435 patients in 4 trials; relative risk 0.55 , 95\% CI 0.31-0.97) showed consistent evidence of efficacy. The authors conclude that antispasmodics, fibre and peppermint oil are more effective than placebo in the treatment of irritable bowel syndrome.

\section{Reference}

Ford AC et al (2008) Effect of fibre, antispasmodics, and peppermint oil in the treatment of irritable bowel syndrome: systematic review and meta-analysis. BMJ 337:a2313

\section{Multidetector computed tomographic angiography and standard coronary angiography in subjects with suspected obstructive coronary artery disease}

Positive and negative predictive values of multidetector computed tomographic angiography suggest that it cannot currently substitute conventional coronary angiography.

A group of international researchers performed a multicenter trial to asses the accuracy of $0.5-\mathrm{mm}$ multidetector 
computed tomographic (CT) angiography (64 detectors), compared with standard coronary angiography in individuals with suspected obstructive coronary artery disease. The patients of this study were recruited in nine different centres and they were evaluated for calcium scoring and examined with multidetector CT angiography before standard coronary angiography. Almost 300 patients (291) who had calcium score not higher than 600 underwent multidetector CT angiography and standard angiography in independent laboratories. Among the patients enrolled, $56 \%$ showed obstructive coronary artery disease (obstructive stenoses were stenoses of at least 50\%). The severity of disease documented by multidetector CT angiography and standard angiography was correlated, as indicated by $r=0.81$. The diagnostic accuracy of multidetector CT angiography in identifying or excluding stenoses of at least $50 \%$ showed an area under the receiver-operating-characteristic curve of 0.93 , a specificity of 0.90 , a sensitivity of 0.85 , a positive predictive value of 0.91 and a negative predictive value of 0.83 . The capacity of multidetector CT angiography in detecting subjects needing subsequent revascularization showed an area under the receiver-operating-characteristic curve of 0.84 , compared to 0.82 for standard angiography.

The authors conclude that, overall, multidetector CT angiography detects in an accurate way both the presence and the grade of severity of obstructive coronary artery disease, but that the positive and negative values of multidetector computed tomographic angiography evidence that it cannot currently substitute standard coronary angiography.

\section{Reference}

Miller JM et al (2008) Diagnostic performance of coronary angiography by 64-Row CT. N Engl J Med 359:2324-2336

\section{Rosuvastatin significantly lowers the incidence of clinical cardiovascular episodes in people without hyperlipidemia and with increased high-sensitivity $\mathrm{C}$-reactive protein levels}

Rosuvastatin is able to prevent major vascular events in apparently healthy males and females without hyperlipidemia but with elevated high-sensitivity $C$-reactive protein levels.
Rosuvastatin is a component of the drug class of statins, which are used to manage hypercholesterolemia and to prevent cardiovascular disease. The researchers of the international JUPITER Study Group formulated the hypothesis that individuals with increased high-sensitivity C-reactive protein values, even if without hyperlipidemia, could take benefit from a therapy with statins, given that these drugs are able to reduce not only cholesterol levels but also high-sensitivity C-reactive protein. As a consequence, almost 18,000 $(17,802)$ apparently healthy males and females, who had values of high-sensitivity C-reactive protein of at least $2 \mathrm{mg} / \mathrm{l}$ and LDL inferior to $130 \mathrm{mg} / \mathrm{dl}$, were randomly allocated to $20 \mathrm{mg}$ a day of rosuvastatin or placebo, and followed for the development of major cardiovascular events. This study was interrupted after a median follow up of 1.9 years, since the rates of myocardial infarction (MI), stroke, revascularization of unstable angina, combined end point of MI, stroke or death from cardiovascular disease, and death from any cause were significantly lower in the rosuvastatin group if compared to the placebo group (respectively: 0.17 vs. $0.37, p=0.0002 ; 0.18$ vs. $0.34, p=0.002 ; 0.41$ vs. 0.77 , $p<0.00001 ; 0.45$ vs. $0.85, p<0.00001 ; 1.00$ vs. 1.25 , $p=0.02)$. The group on rosuvastatin showed an increased incidence of diabetes but did not show a significantly higher incidence of cancer or myopathy if compared to the placebo group.

The authors of this international clinical trial conclude that rosuvastatin significantly lowered the incidence of many different major cardio- and cerebro-vascular events in a large group of apparently healthy males and females without hyperlipidemia but with elevated high-sensitivity C-reactive protein levels.

\section{Reference}

Ridker PM et al (2008) Rosuvastatin to prevent vascular events in men and women with elevated C-reactive protein. N Engl J Med 359:2195-2207

Conflict of interest statement The authors declare that they have no conflict of interest related to the publication of this manuscript. 